\title{
Review: antibiotics did not lead to general improvement in upper respiratory tract infections
}

\author{
Arroll B, Kenealy T. Antibiotics versus placebo in the common cold. (Cochrane Review, latest version 8 Apr 98). In: Cochrane Library. \\ Oxford: Update Software.
}

\section{Question}

What is the efficacy and safety of antibiotics for acute upper respiratory tract infections?

\section{Data sources}

Published and unpublished studies were identified by searching Medline (1966-97) with the content terms antibiotics, respiratory tract infections (upper), common cold, and pharyngitis; Embase/Excerpta Medica; the Family Medicine Database (Canadian College of Family Physicians Library); and the Cochrane Clinical Trials register. Bibliographies of relevant studies, review articles, and textbook chapters were reviewed and primary investigators of studies were contacted.

\section{Study selection}

Randomised controlled trials were selected if they included patients with acute upper respiratory tract infection (with symptoms including rhinitis, sore throat, cough, or productive sputum) who were allocated to antibiotics or placebo and if outcomes included resolution of symptoms of nasopharyngeal inflammation, global ratings of health, or adverse effects. Studies were excluded if $>5 \%$ of patients tested positive for streptococcal infection; patients had a known bacterial diagnosis, bronchitis, or history of serious illness; if antibiotics were given prophylactically; or if the process of randomisation was questionable.

\section{Data extraction}

Data were extracted on study design, participants, intervention, and outcomes. Studies were assessed for methodological quality in terms of randomisation, co-interventions, follow up, and blinding.

\begin{abstract}
Main results
7 trials were included in the analysis. Study drugs included tetracycline, penicillin, ampicillin, amoxycillin and amoxycillin with clavulinic acid, erythromycin, and co-trimoxazole. Metaanalysis of 5 trials $(n=1935)$ using a fixed effects model found no difference between antibiotics and placebo for general improvement at 1 to 7 days (weighted event rates 51.2\% v $52.5 \%, \mathrm{p}=0.43\}^{*}$. Meta-analysis of 4 studies $(\mathrm{n}=1908)$ using a random effects model (because of significant heterogeneity) found a non-significant increase in adverse effects among patients who received antibiotics compared with those who received placebo \{weighted event rates $9.7 \%$ v $3.6 \%, \mathrm{p}=0.11$ \}* 1 study examined nasal discharge and found a modest benefit at 8 days. Another study found a significant benefit for antibiotics in a small subset of patients with positive nasopharyngeal aspirates for 1 of 3 respiratory pathogens (Haemophilus influenzae, Moraxella catarrhalis, and Streptococcus pneumoniae).
\end{abstract}

\section{Conclusion}

Among patients with acute upper respiratory tract infection, antibiotics have no benefit for general improvement compared with placebo, and are associated with a non-significant increase in adverse effects.

\section{*Calculated from data in article.}

\section{Source of funding: no external funding.}

For correspondence: Dr B Arroll, General Practice, University of Auckland, Private Bag 92019, Auckland, New Zealand. Fax +6493737006.

A modified version of this abstract appears in Evidence-Based Medicine.

\section{Commentary}

For several years, healthcare providers have been prescribing fewer antibiotics for the common cold, based on the fact that the aetiology of this frequently diagnosed illness is viral. This review by Arroll and Kenealy substantiates this practice and can be used to inform patients as to why antibiotics are not indicated. The findings of this review have been confirmed by a review by Fahey et al (see abstract, p 77) which concluded that antibiotic treatment of children with upper respiratory tract infection was not supported by current evidence.

Arroll and Kenealy did a comprehensive search of the literature using several electronic databases, reviewed bibliographies of relevant publications, and contacted authors. The authors state that further research is needed to determine the effect of antibiotics on clear or purulent nasal discharge and the role of pathogenic bacteria in upper respiratory tract infections, given the conflicting results of studies in these areas.

Nurses can use the information from this study to educate patients, as well as colleagues for whom prescribing practices may be well established. The decision as to when antibiotic treatment is appropriate is in the healthcare provider's realm, and patient expectations may con- flict with the current research evidence. Through sharing of the evidence with patients and including them in treatment decisions, the practitioner and patient can shift their focus to effective symptom relief measures.

\section{Jo Ann Nicoteri, RN, CS, CRNP, MS Family Nurse Practitioner/Faculty University of Scranton Scranton, Pennsyluania, USA}

1 Fahey T, Stocks N, Thomas T. Systematic review of the treatment of upper respiratory tract of the treatment of upper respiratory tract
infection. Arch Dis Child 1998 Sep;79:225-30. 